Research, Society and Development, v. 10, n. 2, e22110212588, 2021

(CC BY 4.0) | ISSN 2525-3409 | DOI: http://dx.doi.org/10.33448/rsd-v10i2.12588

\title{
Análise da eficácia de ferramentas educativas no conhecimento dos educadores escolares sobre o manejo das avulsões dentárias: uma revisão integrativa
}

\author{
Analysis of the effectiveness of educational tools in the knowledge of school educators about the \\ management of dental avulsions: an integrative review \\ Análisis de la efectividad de las herramientas educativas en el conocimiento de los educadores \\ escolares sobre el manejo de las avulsiones dentales: una revisión integradora
}

Recebido: 02/02/2021 | Revisado: 03/02/2021 | Aceito: 04/02/2021 | Publicado: 13/02/2021

\begin{abstract}
Nilton Freitas Medrado Filho
ORCID: https://orcid.org/0000-0003-1090-4727 Universidade do Estado do Rio Grande do Norte, Brasil

E-mail: niltonfreitasfilho@ hotmail.com

Hanna Marrie Dantas Raposo da Câmara

ORCID: https://orcid.org/0000-0002-5947-1435 Universidade do Estado do Rio Grande do Norte, Brasil

E-mail: marrie.hanna219@gmail.com

Georgia Costa de Araújo Souza

ORCID: https://orcid.org/0000-0002-3342-2627 Universidade do Estado do Rio Grande do Norte, Brasil

E-mail: georgia_odonto@yahoo.com.br

Ana Clara Soares Paiva Tôrres

ORCID: https://orcid.org/0000-0002-7525-3171 Universidade do Estado do Rio Grande do Norte, Brasil E-mail: clarasoares@uern.br

Patrícia Bittencourt Dutra dos Santos

ORCID: https://orcid.org/0000-0003-3308-6376 Universidade do Estado do Rio Grande do Norte, Brasil E-mail: patriciabittencourt@uern.br
\end{abstract}

\begin{abstract}
Resumo
O objetivo foi analisar a efetividade das ferramentas educacionais no conhecimento de educadores escolares sobre o manejo das avulsões dentárias, buscando estabelecer uma relação com as principais condutas iniciais e investigar informações que são repassadas para este público. Trata-se de uma revisão integrativa, cuja coleta de dados foi realizada a partir de fontes primárias, por meio de levantamento bibliográfico nas bases de dados PubMed, Web of Science, SCOPUS, LILACS, BBO e SciELO, no período de outubro a novembro de 2020. Dentre os 461 achados e após utilização de filtros, leitura de títulos e resumos, incluíram-se 9 estudos para compor a revisão. Os resultados do estudo revelaram que a participação do professor escolar diante de uma situação de urgência é fundamental para prestar bons cuidados às crianças, pois a qualidade destes primeiros socorros irá afetar diretamente o prognóstico à longo prazo dos dentes avulsionados. Para isso, métodos de instruções educacionais foram aplicados, tais como: seminários, palestras, pôsteres, divulgação de aplicativo e posteriormente aplicação de questionário para avaliar se os conhecimentos dos educadores escolares aumentaram. Após a instrução dada por profissionais ou estudantes de Odontologia, houve melhora quanto ao nível de conhecimento sobre o meio de transporte do dente, sendo o leite citado em mais de 50\% dos estudos e quanto aos métodos de limpeza, os educadores optaram por lavar o dente com água ou solução salina. Conclui-se, portanto, que os instrumentos em saúde e os programas de educação intervencionistas aumentaram os conhecimentos dos educadores sobre o manejo das avulsões dentárias.
\end{abstract}

Palavras-chave: Conhecimento; Atitude; Professores de escola; Avulsão de dente.

\begin{abstract}
The objective was to analyze the effectiveness of educational tools in the knowledge of school educators about the management of dental avulsions, seeking to establish a relationship with the main initial behaviors and investigate information that are passed on to this public. This is an integrative review, whose data collection was carried out from primary sources, through bibliographic surveys in the databases PubMed, Web of Science, SCOPUS, LILACS, BBO and SciELO, from October to November 2020. Among the 461 findings and after using filters, reading of titles and abstracts, 9 studies were included to compose the review. The results of the study revealed that the participation of
\end{abstract}


Research, Society and Development, v. 10, n. 2, e22110212588, 2021

(CC BY 4.0) | ISSN 2525-3409 | DOI: http://dx.doi.org/10.33448/rsd-v10i2.12588

school teachers in an emergency situation is essential to provide good care to children, because the quality of these first aid will directly affect the long-term prognosis of avulsed teeth. For this, educational instruction methods were applied, such as: seminars, lectures, posters, dissemination of application and later application of a questionnaire to assess whether the knowledge of school educators has increased. After the instruction given by professionals or dental students, there was an improvement in the level of knowledge about the means of transportation of the tooth, with milk being cited in more than $50 \%$ of the studies and as for cleaning methods, the educators chose to wash the tooth with water or saline solution. It is concluded, therefore, that health tools and interventionist education programs have increased educators' knowledge about the management of tooth avulsions.

Keywords: Knowledge; Attitude; School teachers; Tooth avulsion.

\section{Resumen}

El objetivo fue analizar la eficacia de las herramientas educativas en el conocimiento de los educadores escolares sobre el manejo de las avulsiones dentales, buscando establecer una relación con los principales comportamientos iniciales e investigar la información que se transmite a este público. Se trata de una revisión integradora, cuya recolección de datos se realizó a partir de fuentes primarias mediante un relevamiento bibliográfico en las bases de datos PubMed, Web of Science, SCOPUS, LILACS, BBO y SciELO, entre octubre y noviembre de 2020. Entre los 461 hallazgos y tras el uso de filtros, lectura de títulos y resúmenes, se incluyeron 9 estudios para componer la revisión. Los resultados del estudio revelaron que la participación de los maestros de escuela en una situación de emergencia es esencial para proporcionar una buena atención a los niños, ya que la calidad de estos primeros auxilios afectará directamente al pronóstico a largo plazo de los dientes avulsionados. Para ello, se aplicaron métodos de instrucción educativa, como seminarios, conferencias, carteles, difusión de la aplicación y posterior aplicación de un cuestionario para evaluar si los conocimientos de los educadores escolares han aumentado. Después de la instrucción impartida por profesionales o estudiantes de odontología, hubo una mejora en el nivel de conocimiento sobre los medios de transporte del diente, citándose la leche en más del 50\% de los estudios y, en cuanto a los métodos de limpieza, los educadores optaron por lavar el diente con água o solución salina. Por lo tanto, se puede concluir que las herramientas sanitarias y los programas de educación intervencionista han aumentado los conocimientos de los educadores sobre el manejo de las avulsiones dentales.

Palabras clave: Conocimiento; Actitud; Maestros; Avulsión de diente.

\section{Introdução}

As lesões dentárias traumáticas (LDTs) representam um sério problema de saúde pública entre indivíduos crianças e pré-adolescentes em decorrência da sua alta prevalência (Sá et al., 2012; Silva et al., 2016). Atualmente, correspondem a cerca de $5 \%$ do total de injúrias ocorridas no corpo adulto, mas quando ocorridas em crianças esse percentual é ainda maior, sendo a dentição permanente a mais atingida, especialmente os incisivos superiores (Sá et al., 2012; Bustamante-Hernández, et al., 2020). A maioria das lesões traumáticas em crianças é vista entre os 8 e 11 anos de idade (fase escolar e pré-adolescência). É na fase escolar que acidentes como quedas são comuns e se constituem na principal causa de lesões dentárias traumáticas (Curylofo et al., 2012). Dentre a diversidade de LDTs, é possível observar desde fraturas de pequena magnitude até a avulsão dentária (Demir et al., 2020).

A avulsão dentária é definida como um deslocamento total do dente de seu alvéolo, correspondendo a cerca de 1-11\% de todas as lesões dentárias, atingindo principalmente o sexo masculino (Siviero et al., 2005). Comumente, danos severos aos tecidos pulpares e periodontais são observados após a exarticulação completa do dente, o que pode gerar uma série de complicações pós-reimplante, tais como necrose pulpar, anquilose e reabsorção radicular (Bustamante-Hernández et al., 2020).

A intervenção de urgência em casos de dente avulsionado deve ser realizada no menor tempo possível, uma vez que o prognóstico do dente reimplantado depende de medidas adequadas tomadas no local do acidente (Fouad et al., 2020). Alguns trabalhos relatam a falta de preparo da população, educadores, profissionais do esporte e até mesmo profissionais de saúde frente aos traumatismos dentários (Lima, 2010; Tewari et al., 2020). Devido à faixa etária de 8 a 11 anos ser a mais atingida e ainda aos fatores causais mais frequentemente envolvidos, entende-se que o ambiente escolar infantil é um dos mais associados à ocorrência de lesões dentárias traumáticas (Kallel et al., 2020)

A efetividade da informação na mudança de comportamento de educadores sobre como agir frente à avulsão dentária 
vem sendo investigada. Tem sido sugerida a importância de realização de campanhas educativas esclarecendo um protocolo adequado a ser seguido em tais situações, de modo a garantir a manutenção em longo prazo desse dente. A orientação sobre as condutas de emergência que deverão ser tomadas em casos de avulsão dentária é de suma importância, como forma de favorecer o prognóstico dos dentes traumatizados (Enikawati et al., 2020).

Para tanto, o presente estudo teve como objetivo avaliar a efetividade das ferramentas educacionais no conhecimento de educadores escolares sobre o manejo das avulsões dentárias.

\section{Metodologia}

Este estudo foi desenvolvido de acordo com Pereira et al, (2018), onde se trata de uma pesquisa qualitativa que se relaciona com a variedade de achados e que consiste na interpretação por parte do pesquisador com suas opiniões sobre o fenômeno em estudo, bem como na conclusão e verificação dos dados.

Trata-se de uma revisão integrativa com coleta de dados realizada a partir de fontes primárias, por meio de levantamento bibliográfico na literatura. A questão norteadora da pergunta foi estruturada de acordo com o acrônimo PICO (P: população/pacientes; I: intervenção; C: comparação/controle; O: desfecho/outcome) (Tabela 1), definida como: Ferramentas educacionais são efetivas na orientação de educadores escolares sobre o manejo das avulsões dentárias?

Tabela 1. Estratégia seguindo o acrônimo PICO (População/Intervenção/Comparação e Desfecho).

\begin{tabular}{ccc}
\hline ACRÔNIMO & DEFINIÇÃO & DESCRIÇÃO \\
\hline P & População & Professores escolares \\
\hline I & Intervenção & $\begin{array}{c}\text { Informação dada aos educadores escolares sobre o } \\
\text { manejo das avulsões dentárias }\end{array}$ \\
\hline $\mathbf{C}$ & Comparação & $\begin{array}{c}\text { Educadores escolares que não receberam orientações } \\
\text { sobre o manejo das avulsões dentárias }\end{array}$ \\
\hline $\mathbf{O}$ & Resultado & A correta conduta nos primeiros cuidados após avulsões \\
dentárias
\end{tabular}

Fonte: Autores (2020).

Para o levantamento dos artigos, realizou-se uma busca nas bases de dados: PubMed, Web of Science, SCOPUS, LILACS, BBO e SciELO. Os descritores para a busca dos artigos foram selecionados a partir do DeCS (Descritores em Ciências da Saúde) e MeSH (Medical Subject Heading), sendo estes: Knowledge, Attitude, Awareness, "School Teachers", Faculty e "Tooth Avulsion". Foram utilizados os operadores booleanos "AND" e "OR" para fazer as combinações entre os termos selecionados (Quadro 1). O levantamento dos artigos foi realizado nos meses de outubro e novembro de 2020. 
Quadro 1. Estratégia de busca utilizada nas bases de dados selecionadas.

\begin{tabular}{|c|c|}
\hline $\begin{array}{l}\text { Base de } \\
\text { dado }\end{array}$ & Estratégia utilizada \\
\hline PubMed & $\begin{array}{l}\text { ((Knowledge) OR (Attitude)) OR (Awareness)) AND (School Teachers)) OR } \\
\text { (Faculty)) AND (Tooth Avulsion) }\end{array}$ \\
\hline $\begin{array}{l}\text { Web of } \\
\text { Science }\end{array}$ & $\begin{array}{l}\text { ((Knowledge) OR (Health Knowledge Attitudes Practice) OR (Attitude) OR } \\
\text { (Attitudes)) AND ((School Teachers) OR (Teacher School) OR (School } \\
\text { Teacher) OR (High School Teachers) OR (School Teacher High) OR (School } \\
\text { Teachers High) OR (Teacher High School) OR (Teachers High School) OR } \\
\text { (Middle School Teachers)) AND ((Tooth Avulsion) OR (Avulsion Tooth) OR } \\
\text { (Avulsions Tooth) OR (Tooth Avulsions) OR (Avulsed Tooth) OR (Tooth } \\
\text { Avulsed)) }\end{array}$ \\
\hline SCOPUS & $\begin{array}{l}\text { ((Knowledge) OR (Health Knowledge Attitudes Practice) OR (Attitude) OR } \\
\text { (Attitudes)) AND ((School Teachers) OR (Teacher School) OR (School } \\
\text { Teacher) OR (High School Teachers) OR (School Teacher High) OR (School } \\
\text { Teachers High) OR (Teacher High School) OR (Teachers High School) OR } \\
\text { (Middle School Teachers)) AND ((Tooth Avulsion) OR (Avulsion Tooth) OR } \\
\text { (Avulsions Tooth) OR (Tooth Avulsions) OR (Avulsed Tooth) OR (Tooth } \\
\text { Avulsed)) }\end{array}$ \\
\hline LILACS & $\begin{array}{l}\text { (Knowledge OR Conhecimento) AND (School OR Escolares) AND (Tearchers } \\
\text { OR Professores) AND (Tooth Avulsion OR Avulsão Dentária) }\end{array}$ \\
\hline BBO & $\begin{array}{l}\text { (Knowledge OR Conhecimento) AND (School OR Escolares) AND (Tearchers } \\
\text { OR Professores) AND (Tooth Avulsion OR Avulsão Dentária) }\end{array}$ \\
\hline SciELO & $\begin{array}{l}\text { (Knowledge OR Conhecimento) AND (School OR Escolares) AND (Teachers } \\
\text { OR Professores) AND (Faculty OR Docentes) AND (Tooth OR Dentária) } \\
\text { AND (Avulsion OR Avulsão) }\end{array}$ \\
\hline
\end{tabular}

Fonte: Autores (2020).

Os critérios de inclusão definidos foram estudos com professores escolares, artigos publicados em português, inglês ou espanhol indexados nas bases de dados selecionadas e com texto completo de acesso livre. Como critérios de exclusão: estudos que não apresentassem relação com a temática de interesse, teses, dissertações ou aqueles que não fossem estudos primários.

Após a seleção dos artigos em cada base de dado, os mesmos foram enviados para um gerenciador de referências e software de processamento (Mendeley ${ }^{\circledR}$ Desktop, Elsevier) para melhor coleta das informações. O processo de extração de dados dos artigos que passaram por triagem foi guiado por um documento padrão de fichamento para análise dos estudos.

\section{Resultados}

Foi identificado inicialmente um total de 461 artigos. Destes, 105 foram removidos devido ao fato de serem trabalhos duplicados. Posteriormente, a seleção ocorreu por meio da leitura de título de cada artigo, obtendo-se 72 resultados e excluindo-se 284 textos que não estavam de acordo com os critérios pré-estabelecidos. Em seguida, realizou-se a seleção pelo resumo, onde foram selecionados 53 artigos e excluídos 19. Por fim, dos 53 artigos selecionados para leitura na íntegra, 44 foram excluídos. A amostra final do presente trabalho foi composta por 9 estudos primários que cumpriram todos os critérios de elegibilidade (Figura 1), publicados entre 2006 e 2020. 
Research, Society and Development, v. 10, n. 2, e22110212588, 2021

(CC BY 4.0) | ISSN 2525-3409 | DOI: http://dx.doi.org/10.33448/rsd-v10i2.12588

Figura 1. Diagrama de fluxo PRISMA de estudos selecionados.

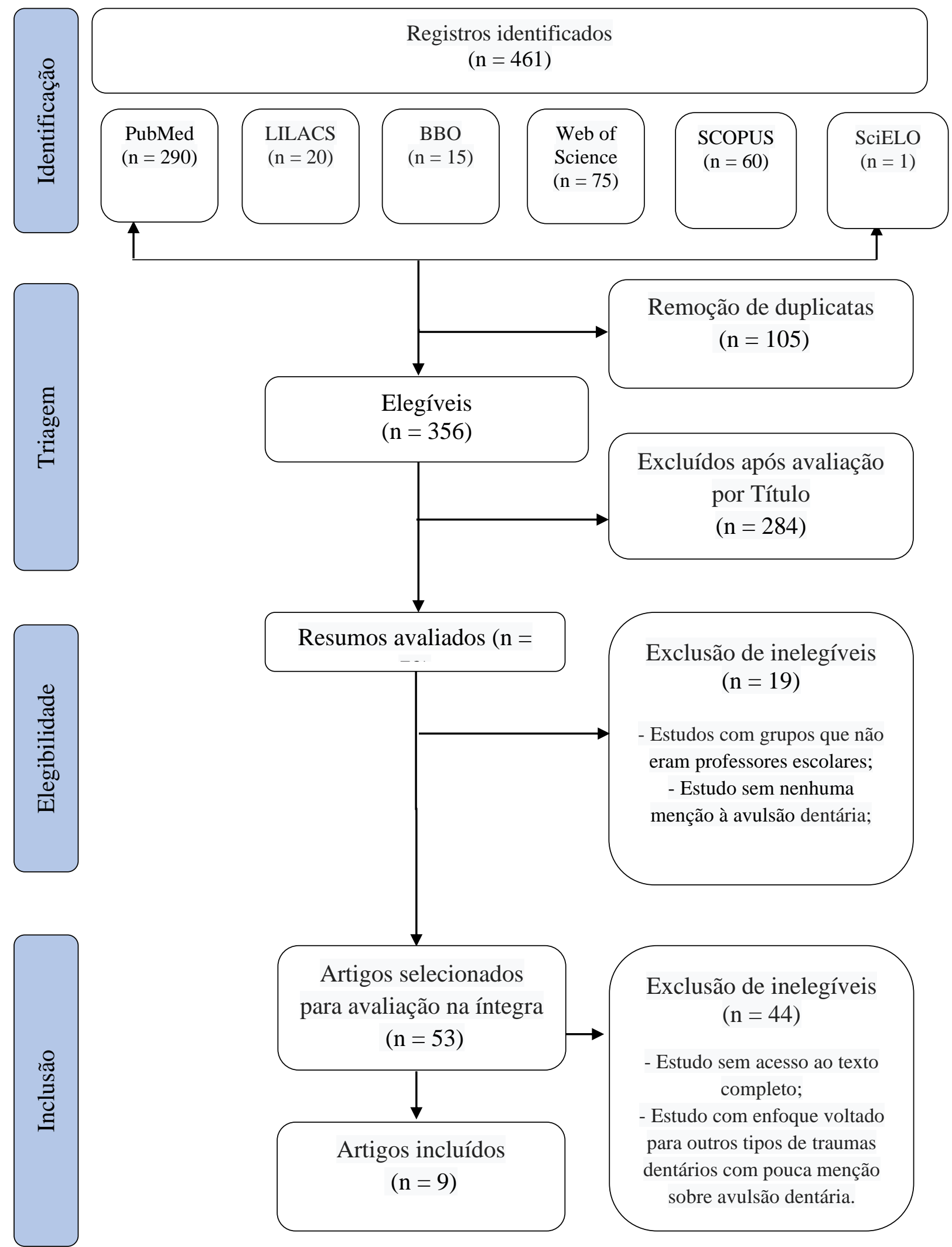

Fonte: Autores (2020).

Todos os estudos incluídos tiveram aplicação de questionário ou formulário sobre o conhecimento de educadores 
Research, Society and Development, v. 10, n. 2, e22110212588, 2021

(CC BY 4.0) | ISSN 2525-3409 | DOI: http://dx.doi.org/10.33448/rsd-v10i2.12588

escolares acerca do manejo das avulsões dentárias. No total, foram 934 professores dos nove estudos selecionados, com a amostra variando de 50 (Kharpate et al., 2020) a 226 professores (Holan et al., 2006). O tipo de professor variou do ensino primário/fundamental (Mori et al., 2007) ao ensino secundário (Kharpate et al., 2020). Os principais aspectos metodológicos e resultados observados podem ser vistos no Quadro 2.

Quadro 2. Síntese dos artigos intervencionistas incluídos na revisão integrativa.

\begin{tabular}{|c|c|c|c|c|c|}
\hline Autores/ano & $\begin{array}{l}\text { País/ } \\
\text { Cidade }\end{array}$ & $\begin{array}{c}\text { Tamanho } \\
\text { da } \\
\text { Amostra }\end{array}$ & $\begin{array}{c}\text { Tipo de } \\
\text { Professor }\end{array}$ & $\begin{array}{c}\text { Tipo de } \\
\text { Intervenção }\end{array}$ & Desfecho \\
\hline $\begin{array}{l}\text { HOLAN et } \\
\text { al., } 2006\end{array}$ & $\begin{array}{l}\text { Israel, } \\
\text { Jerusalé } \\
\mathrm{m}\end{array}$ & 226 & $\begin{array}{l}\text { Professores de } \\
\text { educação física }\end{array}$ & Seminário & $\begin{array}{l}\text { O seminário ministrado aos professores de } \\
\text { educação física (em } 2003 \text { e 2004) acerca do manejo } \\
\text { emergencial dos dentes avulsionados mostrou um } \\
\text { relevante aumento no desempenho dos educadores. } \\
\text { Quando questionados se eles iriam reimplantar um } \\
\text { dente avulsionado (se necessário), } 19 \% \text { (24 de 126) } \\
\text { dos entrevistados em } 2003 \text { e } 53 \% \text { ( } 53 \text { de 100) dos } \\
\text { entrevistados em } 2004 \text { forneceram um resultado } \\
\text { positivo em relação à resposta. }\end{array}$ \\
\hline $\begin{array}{l}\text { MORI et al., } \\
2007\end{array}$ & $\begin{array}{l}\text { Brasil, } \\
\text { Adamanti } \\
\text { na-SP }\end{array}$ & 80 & $\begin{array}{l}\text { Professores do } \\
\text { ensino primário }\end{array}$ & $\begin{array}{l}\text { Campanha } \\
\text { informativa com uso } \\
\text { de banners e folders }\end{array}$ & $\begin{array}{l}\text { A campanha informativa mostrou eficácia, onde } \\
100 \% \text { dos entrevistados responderam à importância } \\
\text { da emergência dos procedimentos em caso de } \\
\text { avulsão dentária. Além disso, } 81,25 \% \text { dos } \\
\text { entrevistados responderam que armazenariam o } \\
\text { dente avulsionado em algum meio de } \\
\text { armazenamento adequado, sendo o leite o mais } \\
\text { citado }(84,61 \%) \text {. }\end{array}$ \\
\hline $\begin{array}{l}\text { AL-ASFOUR } \\
\text { et al., } 2008\end{array}$ & $\begin{array}{l}\text { Kuwait, } \\
\text { Kuwait }\end{array}$ & 85 & $\begin{array}{l}\text { Professores de } \\
\text { escolas } \\
\text { intermediárias } \\
\text { (crianças de } 10 \\
\text { a } 14 \text { anos) }\end{array}$ & Palestra & $\begin{array}{l}\text { O nível de conhecimentos dos professores acerca } \\
\text { da avulsão e reimplante aumentou } \\
\text { exponencialmente em todos os pontos abordados } \\
\text { nos questionários após a palestra fornecida. O } \\
\text { conhecimento geral de avulsão dentária e o } \\
\text { reimplante melhorou de } 39 \% \text { para } 97 \% \text {. }\end{array}$ \\
\hline $\begin{array}{l}\text { TRIGUEIRO } \\
\text { et al., } 2015\end{array}$ & $\begin{array}{l}\text { Brasil, } \\
\text { João } \\
\text { Pessoa- } \\
\text { PB }\end{array}$ & 84 & $\begin{array}{l}\text { Professores do } \\
\text { ensino } \\
\text { fundamental }\end{array}$ & Palestra & $\begin{array}{l}\text { Antes da palestra, apenas } 2,8 \% \text { e } 13,3 \% \\
\text { transportariam o dente avulsionado no leite e na } \\
\text { saliva. Sobre possibilidade de reimplante do dente } \\
\text { avulsionado, a alternativa "sim" elevou de } 27,7 \% \\
\text { para } 72,3 \% \text { e a resposta "talvez" reduziu de } 75,6 \% \\
\text { para } 24,4 \% \text {, caracterizando mais segurança por } \\
\text { parte do professor. }\end{array}$ \\
\hline $\begin{array}{l}\text { AL- } \\
\text { MUSAWI et } \\
\text { al., } 2016\end{array}$ & $\begin{array}{l}\text { Kuwait, } \\
\text { Kuwait }\end{array}$ & 87 & $\begin{array}{l}\text { Professores do } \\
\text { ensino } \\
\text { fundamental e } \\
\text { médio }\end{array}$ & $\begin{array}{l}\text { Aplicativo para } \\
\text { smartphone e } \\
\text { palestra }\end{array}$ & $\begin{array}{l}\text { As intervenções utilizadas no estudo aumentaram o } \\
\text { conhecimento da gestão da avulsão dentária entre } \\
\text { os professores. O aumento foi observado nas } \\
\text { seguintes áreas: manipulação do dente no solo } \\
\text { (16\% a } 96,8 \%) \text {; manutenção do dente durante a } \\
\text { limpeza }(19,1 \% \text { a } 62,5 \%) \text {; e sobre o melhor meio } \\
\text { de armazenamento (10,1\% a } 59.3 \%) \text {. }\end{array}$ \\
\hline $\begin{array}{l}\text { TARANATH } \\
\text { et al., } 2017\end{array}$ & $\begin{array}{l}\text { Índia, } \\
\text { East } \\
\text { Madurai } \\
\text { Panchaya } \\
\text { t }\end{array}$ & 214 & $\begin{array}{l}\text { Professores do } \\
\text { ensino primário }\end{array}$ & $\begin{array}{l}\text { Palestra + } \\
\text { demonstração } \\
\text { prática }\end{array}$ & $\begin{array}{l}\text { O nível de conhecimento de } 70,83 \% \text { dos } \\
\text { professores do ensino primário estava na categoria } \\
\text { "muito boa" depois da ferramenta de educação em } \\
\text { saúde, que inicialmente era nula. Da mesma forma, } \\
\text { o nível de atitude também aumentou drasticamente } \\
\text { com } 0,90 \% \text { inicialmente na categoria "muito boa", } \\
\text { e depois da ferramenta de educação, foi de } 41,20 \% \text {. }\end{array}$ \\
\hline $\begin{array}{l}\text { KATTHIKA } \\
\text { et al., } 2020\end{array}$ & $\begin{array}{l}\text { Indonésia } \\
\text {, Jakarta }\end{array}$ & 54 & $\begin{array}{l}\text { Professores do } \\
\text { ensino primário }\end{array}$ & Vídeo animado & $\begin{array}{l}\text { A medição da pontuação total do conhecimento dos } \\
\text { professores foi realizada antes e depois de assistir a } \\
\text { um vídeo animado. } 100 \% \text { dos sujeitos } \\
\text { demonstraram estatisticamente aumento } \\
\text { significativo no total da pontuação de } \\
\text { conhecimento depois de assistir ao vídeo. }\end{array}$ \\
\hline KHARPATE & Índia, & 50 & Professores & Orientações & O conhecimento de $98 \%$ dos professores aumentou \\
\hline
\end{tabular}


Research, Society and Development, v. 10, n. 2, e22110212588, 2021

(CC BY 4.0) | ISSN 2525-3409 | DOI: http://dx.doi.org/10.33448/rsd-v10i2.12588

\begin{tabular}{|l|l|l|l|l|}
\hline et al., 2020 & Wardha & $\begin{array}{l}\text { ensino primário } \\
\text { e secundário }\end{array}$ & $\begin{array}{l}\text { após a atividade enquanto o conhecimento de 2\% } \\
\text { dos professores foi o mesmo que antes. O estudo } \\
\text { evidenciou que houve melhora na consciência e } \\
\text { atitude para gerir o traumatismo dentário e a lesão } \\
\text { por avulsão dos dentes na escola. }\end{array}$ \\
\hline $\begin{array}{l}\text { ENIKAWATI } \\
\text { et al., 2020 }\end{array}$ & $\begin{array}{l}\text { Indonésia } \\
\text { Jacarta }\end{array}$ & 54 & $\begin{array}{l}\text { Professores do } \\
\text { ensino primário }\end{array}$ & $\begin{array}{l}\text { Pôster informativo } \\
\text { Em geral, a pontuação mediana antes da educação } \\
\text { era de 5 pontos (variação: 1-10 pontos). Após a } \\
\text { educação, a pontuação mediana foi de 10 10 pontos } \\
\text { (variação: 4-14 pontos). As estatísticas mostraram } \\
\text { uma diferença significativa entre antes e depois da } \\
\text { implantação do pôster. }\end{array}$ \\
\hline
\end{tabular}

Fonte: Autores (2020).

\section{Discussão}

Os estudos incluídos nesta revisão foram selecionados de acordo com a pergunta norteadora deste trabalho, que diz respeito à eficácia das ferramentas educacionais sobre o manejo das avulsões dentárias para educadores escolares. Foram incluídos apenas estudos intervencionistas, nos quais métodos de instruções educacionais foram aplicados, tais como: seminários, palestras, pôsteres, divulgação de aplicativo e posteriormente aplicação de questionário. Esse trabalho também incluiu os estudos primários em ambiente escolar com professores do ensino primário e secundário no geral e estudos especificamente com professores de educação física destes mesmos graus de ensino (Holan et al., 2006). Para este último grupo, recomenda-se que a atenção seja redobrada, pois as atividades esportivas são as razões mais comuns para trauma dentário entre crianças (Granville-Garcia et al., 2007; Pagliarin et al., 2011).

Alguns estudos mostram que a maioria dos professores acreditam que a limpeza com água é o procedimento correto a ser realizado após a avulsão dentária (Taranath et al., 2017; Kharpate et al., 2020). Enquanto que em outros relatos, professores sugerem o uso de álcool, desinfetante ou solução antisséptica para armazenamento do dente (Enikawati et al., 2020). Entretanto, para um bom prognóstico do reimplante dentário o leite é o mais desejável tanto para a limpeza, quanto para o armazenamento devido ao pH e osmolaridade não serem prejudiciais às células periodontais (Trigueiro et al., 2015). Nesse sentido, a utilização de instrumentos de educação, como questionários ou palestras parecem ser capazes de alterar a conduta dos professores frente à escolha dos meios de armazenamento dentário, bem como em relação ao correto manuseio do dente avulsionado (Trigueiro et al., 2015; Taranath et al., 2017). Nesse sentido, houve melhora considerável quanto ao conhecimento sobre o meio de transporte do dente, sendo o leite citado em mais de 50\% dos estudos (Mori et al., 2007; Al-Asfour et al., 2008; Trigueiro et al., 2015; Taranath et al., 2017; Kharpate et al., 2020). Quanto aos métodos de limpeza, os educadores optaram por lavar o dente com água ou solução salina (Kharpate et al., 2020).

Ferramentas de ensino envolvendo palestra com ou sem demonstração prática e com média de tempo 20 minutos acarretaram um aumento significativo do conhecimento de professores, de modo que após a palestra, o manuseio pela porção da coroa do dente avulsionado foi a parte preferível, a limpeza do dente avulsionado foi considerada necessária, a manutenção extra-alveolar dos dentes avulsionados tiveram uma maior consciência por partes dos educadores e os meios de armazenamento mudaram para uma condição mais apropriada para cicatrização periodontal e pulpar, como o leite e água (Taranath et al., 2017; Kharpate et al., 2020; Trigueiro et al., 2020).

Diversos trabalhos mostram que os professores não detêm conhecimentos sobre o tempo extra-alveolar no prognóstico de tratamento (Al-Asfour et al., 2008; Blakytny et al., 2001; Prasanna et al., 2011; Shamarao et al., 2014; Pratyusha et al., 2015; Pagliarin et al., 2011). A vitalidade e a permanência do dente após o reimplante depende diretamente do tempo decorrido entre o trauma e o atendimento odontológico, entretanto, muitos educadores desconhecem essa informação (Taranath et al., 
2017; Kharpate et al., 2020). Diante disso, algumas ferramentas de ensino foram testadas e efetivamente apresentaram resultados positivos neste quesito. Como exemplo, o aplicativo de smartphone Dental Trauma App, associado ou não a palestras informativas (Al-Musawi et al., 2016). Em adição, métodos convencionais como apresentações orais ou demonstrações práticas também resultaram em aumento significativo no conhecimento dos professores sobre o tema (AlAsfour et al., 2008; Taranath et al., 2017). Entende-se então que ações de educação e informação em saúde são excelentes oportunidades para um encontro direto entre o palestrante e o grupo receptor, havendo a possibilidade de levantar dúvidas e discutir assuntos. Além disso, o palestrante pode obter um retorno imediato se a mensagem foi entendida ou não e receber recomendações quanto ao tipo de informação que deve ser desenvolvida (Al-Asfour et al., 2008).

Em relação ao tratamento considerado de escolha, o reimplante dentário é o mais indicado, tendo em vista que é considerado uma das condutas mais conservadoras na Odontologia, já que preserva o elemento dentário, diminui a necessidade de trabalhos reabilitadores e ainda reduz o impacto psicológico decorrente da perda imediata do dente (Trigueiro et al., 2015). Estudos como o de Berti et. al, (2011) e Shamarao et. al., (2014), relatam através dos seus resultados que uma pequena parcela dos professores escolares (32,89\%) teve acesso a curso de primeiros-socorros, e dentre estes, uma parcela ainda menor (14\%) obteve informações sobre o tema avulsão. E por essa razão, a maioria dos professores não se sente segura para reposicionar um dente avulsionado no alvéolo dentário (Berti et al., 2011; Blakytny et al., 2001; Curylofo et al., 2012; Khan et al., 2020; Prathyusha et al., 2015).

Taranath et al., (2017), alerta que é importante estar ciente de que o reimplante não deve ser realizado quando um dente decíduo for avulsionado, pois há risco de lesão do germe sucessor permanente e dano à dentição. Entretanto, um estudo tem mostrado que a maioria dos professores, já estavam cientes de que um dente decíduo avulsionado não requer reimplante, mesmo sem terem recebido instruções educacionais a respeito (Al-Musawi et al., (2016).

No tocante aos materiais educativos, pôsteres, palestras e seminários podem ser utilizados durante os programas de capacitação de professores para aumentar o conhecimento desses profissionais a respeito da avulsão dentária. Assim, informações apresentadas de forma clara e simples podem ser uma das maneiras mais eficazes para melhorar os resultados do tratamento de dentes permanentes avulsionados (Kaur et al., 2012). Além desses, o uso de aplicativos na instrução de gerenciamento das avulsões dentárias amplia a aprendizagem pelo fato de se qualificar como uma metodologia ativa, que requer participação direta dos usuários, o que pode melhorar a retenção de informações em curto e longo prazo (Al-Musawi et al., 2016).

Apenas nove estudos cumpriram os critérios de inclusão estabelecidos, uma vez que grande parcela de estudos relacionados ao conhecimento de professores sobre avulsões dentárias eram observacionais. Nessa direção, sugerem-se mais estudos intervencionistas avaliando a efetividade dos programas de educação em saúde com enfoque para as avulsões dentárias em âmbito escolar.

\section{Conclusão}

Ferramentas de educação em saúde testadas a partir dos estudos aumentaram consideravelmente o conhecimento dos educadores em relação ao manejo pós-traumático em casos de avulsão dentária. Destaca-se, ainda, a importância de intensificar-se cada vez mais as práticas de educação em saúde voltadas para o manejo desse tipo de LDT entre professores, pois as necessidades de informação dos profissionais são importantes para o aprimoramento do conhecimento, o que acaba minimizando possíveis implicações para o padrão futuro de procura por atendimento odontológico.

Dado o exposto, é imprescindível que mais estudos intervencionistas sejam realizados, a fim de caracterizar as reais 
Research, Society and Development, v. 10, n. 2, e22110212588, 2021

(CC BY 4.0) | ISSN 2525-3409 | DOI: http://dx.doi.org/10.33448/rsd-v10i2.12588

condições dos professores escolares e necessidades desses profissionais, bem como acompanhá-los ao longo do tempo para perceber melhora ou piora do conhecimento acerca da avulsão dentária.

\section{Referências}

Al-Asfour, A., Andersson, L., \& Al-Jame, Q. (2008). School teachers' knowledge of tooth avulsion and dental first aid before and after receiving information about avulsed teeth and replantation. Dental Traumatology, 24(1), 43-49.

Al-Musawi, A., Al-Sane, M., \& Andersson, L. (2017). Smartphone App as an aid in the emergency management of avulsed teeth. Dental Traumatology, 33(1), $13-18$.

Berti, M., Furlanetto, D. L. C., \& Refosco, M. Z. (2011). Avaliação do conhecimento de professores do ensino fundamental sobre o tema avulsão dentária. Pesquisa Brasileira Em Odontopediatria e Clinica Integrada, 11(3), 381-386.

Blakytny, C., Surbuts, C., Thomas, A., \& Hunter, M. L. (2001). Avulsed permanent incisors: Knowledge and attitudes of primary school teachers with regard to emergency management. International Journal of Paediatric Dentistry, 11(5), 327-332.

Bustamante-Hernández, N., Amengual-Lorenzo, J., Fernández-Estevan, L., Zubizarreta-Macho, A., da Costa, C. G. M., \& Agustín-Panadero, R. (2020). What can we do with a dental avulsion? A multidisciplinary Clinical Protocol. Journal of Clinical and Experimental Dentistry, $12(10)$, e991-e998.

Curylofo, P. A., Lorencetti, K. T., \& Silva, S. R. C. (2012). Avaliação do conhecimento de professores sobre avulsão dentária. Arquivos Em Odontologia, 48(3), 175-180.

Demir, P., Guler, C., Kizilci, E., \& Keskin, G. (2020). Survival of avulsed permanent incisors in children following delayed replantation. Niger J Clin Pract, $23,631-637$.

Enikawati, M., Fauziah, E., \& Budiardjo, S. B. (2020). Effect of First Aid Management of Dental Avulsion Posters on Primary School Teachers’ Knowledge. Pesquisa Brasileira Em Odontopediatria e Clínica Integrada, 20.

Fouad, A. F., Abbott, P. V., Tsilingaridis, G., Cohenca, N., Lauridsen, E., Bourguignon, C., O’Connell, A., Flores, M. T., Day, P. F., Hicks, L., Andreasen, J. O., Cehreli, Z. C., Harlamb, S., Kahler, B., Oginni, A., Semper, M., \& Levin, L. (2020). International Association of Dental Traumatology guidelines for the management of traumatic dental injuries: 2. Avulsion of permanent teeth. Dental Traumatology, 36(4), 331-342.

Granville-Garcia, A. F., Lima, E. M., Santos, P. G., \& Menezes, V. A. A. (2007). Avaliação do Conhecimento dos Professores de Educação Física de CaruaruPE Sobre Avulsão-Reimplante Evaluation of the Knowledge of Physical Education Teachers from the City of. Pesq Bras Odontoped Clin Integr, João Pessoa, $7(1), 15-20$.

Holan, G., Cohenca, N., Brin, I., \& Sgan-Cohen, H. (2006). An oral health promotion program for the prevention of complications following avulsion: The effect on knowledge of physical education teachers. Dental Traumatology, 22(6), 323-327.

Kallel, I., Douki, N., Amaidi, S., \& Ben Amor, F. (2020). The Incidence of Complications of Dental Trauma and Associated Factors: A Retrospective Study. International Journal of Dentistry, 2020.

Katthika, V. K., Fauziah, E., \& Budiardjo, S. B. (2020). Animated video for increasing primary school teachers' knowledge regarding first aid management of dental avulsion. Brazilian Dental Science, 23(4), 1-7.

Kaur, H., Kaur, S., \& Kaur, H. (2012). Prehospital emergency management of avulsed permanent teeth: Knowledge and attitude of school teachers. Indian Society for Dental Research, 23(4), 556.

Khan, A., Goyal, A., Somaiya, V., Rathesh, A., Sathiyamoorthy, J., Larkin, K., Currell, S. D., \& Nimmo, A. J. (2020). Knowledge of Australian primary education providers towards dental avulsion injuries: a cross-sectional study. Australian Dental Journal, 65(1), 46-52.

Kharpate, S., Rathi, N., Gomase, P. V., Baliga, S., \& Thosar, N. (2020). Appraisal of Awareness and Attitude of School Teachers towards Emergency Management of Dental Trauma and Tooth Avulsion Replantation. Journal of Evolution of Medical and Dental Sciences, 9(40), 2951-2954.

Lima, D. C. de. (2010). Traumatismo alvéolo-dentário: prevalência em crianças e conhecimento de educadores do ensino fundamental. In - Araçatuba.

Mori, G. G., Castilho, L. R., Nunes, D. C., Turcio, K. H. L., \& Molina, R. O. (2007). Avulsion of permanent teeth: Analysis of the efficacy of an informative campaign for professionals from elementary schools. Journal of Applied Oral Science, 15(6), 534-538.

Mori, G. G., Turcio, K. H. L., Borro, V. P. B., \& Mariusso, Â. M. (2007). Evaluation of the knowledge of tooth avulsion of school professionals from Adamantina, São Paulo, Brazil. Dental Traumatology, 23(1), 2-5.

Pagliarin, C. L., Zenkner, C. L., \& Barletta, F. B. (2011). Knowledge of physical education teachers about emergency management of tooth avulsion. Stomatos, 17(33), 32-42.

Pereira, A. S., et al. (2018). Metodologia da pesquisa científica. UFSM. https://repositorio.ufsm.br/bitstream/handle/1/15824/Lic_Computacao_MetodologiaPesquisa-Cientifica.pdf?sequence $=1$.

Prasanna, S., Giriraju, A., \& Narayan, N. L. (2011). Knowledge and Attitude of Primary School Teachers toward Tooth Avulsion and Dental First Aid in Davangere City: A Cross-sectional Survey. International Journal of Clinical Pediatric Dentistry, 4(3), $203-206$. 
Research, Society and Development, v. 10, n. 2, e22110212588, 2021

(CC BY 4.0) | ISSN 2525-3409 | DOI: http://dx.doi.org/10.33448/rsd-v10i2.12588

Prathyusha, P., Harshini, T., Haripriya, B., Pramod, I. J., Swathi, K., \& Samyuktha, C. L. (2015). Knowledge and Awareness Regarding Avulsion and Its Immediate Treatment in School Teachers in Bangalore City (South). Journal of International Oral Health: JIOH, 7(8), $93-97$.

Sá, M. A. B., Oliveira, S. K. M., Pereira, M. M., Barbosa, G. E. F., \& Freitas, D. A. (2012). Traumatismo dentário em crianças. EFDeportes.Com, Revista Digital, 17(171).

Shamarao, S., Tikare, S., Ajagannanavar, S., Haridas, R., Jain, J., \& Kalappa, A. (2014). Knowledge and attitude regarding management of tooth avulsion injuries among school teachers in rural India. Journal of International Society of Preventive and Community Dentistry, 4(4), 44.

Silva, M. L. C. A., Musse, J. O., Almeida, A. H. V., Marques, J. A. M., \& Costa, M. C. O. (2016). Injúrias dentárias traumáticas em crianças e adolescentes vítimas de violência periciadas no Instituto Médico Legal de Feira de Santana, Bahia. Revista Da Faculdade de Odontologia - UPF, 21(1), 31-36.

Taranath, M., Senaikarasi, R., \& Manchanda, K. (2017). Assessment of knowledge and attitude before and after a health education program in East Madurai primary school teachers with regard to emergency management of avulsed teeth. Journal of Indian Society of Pedodontics and Preventive Dentistry, 35(1), 6367.

Trigueiro, M., Costa, M. M. T. M., Souza, I. C. G., Leitão, G. L. N. C., \& Ortega, A. O. L. (2015). Avulsão dentária: efeito da informação na mudança de comportamento dos professores do ensino fundamental TT - Dental avulsion: information effects in change behavior of elementary school teachers. Robrac, 24(69), 57-61. 